\title{
ADJOINT FUNCTORS INDUCED BY ADJOINT LINEAR TRANSFORMATIONS
}

\author{
PAUL H. PALMQUIST
}

\begin{abstract}
Adjoint linear transformations between Hilbert spaces or, more generally, between dual systems of topological vector spaces induce contravariant functors which are adjoint on the right-essentially a Galois connection between the posets of subsets (or subspaces) of the spaces. Modulo scalars the passage from linear maps to functors is one-to-one; indeed, modulo scalars, two linear transformations are adjoint (hence both are weak continuous) if and only if the induced functors are adjoint.
\end{abstract}

In a symposium honoring Marshall Stone, Saunders Mac Lane explored a formal analogy of adjoint linear operators on a Hilbert space to adjoint functors between categories [3, pp. 230-232], [4, p. 103]. In contrast, this note singles out a more direct connection: adjoint operators yield adjoint functors.

First, we state the theorem and corollary for Hilbert spaces. Second, as suggested by the referee, we generalize the theorem to dual systems [5, pp. 123, 128] of topological vector spaces and (weak continuous) linear transformations. $\$ 3$ contains remarks and definitions. $\$ 4$ proves the general theorem.

1. Adjoints in Hilbert space. Consider two complex Hilbert spaces $H_{0}$ and $H_{1}$, and two maps (=linear transformations) $T_{0}: H_{0} \rightarrow H_{1}$ and $T_{1}$ : $H_{1} \rightarrow H_{0}$. (For notation, let $i, j=0$ or 1 , and $i \neq j$ in the sequel.) The inner product on $H_{i}$ is denoted by $(,)_{i}$. Let $\mathscr{C}_{i}$ be the (small) category whose set of objects $\left|\mathscr{C}_{i}\right|$ consists of all subsets of $H_{i}$ and whose only arrows are inclusions $\left(X \rightarrow Y\right.$ in $\mathscr{C}_{i}$ iff $\left.X \subseteq Y \subseteq H_{i}\right)$. For $A$ in $\left|\mathscr{C}_{i}\right|$, let

$$
D_{i} A=\left\{b \in H_{j} \mid\left(T_{i} a, b\right)_{j}=0, \forall a \in A\right\} .
$$

THEOREM 1. With $T_{i}$ and $D_{i}$ as above the following statements are true.

(i) This yields a contravariant functor $D_{i}$ from $\mathscr{C}_{i}$ to $\mathscr{C}_{j}$.

Received by the editors January 2, 1973 and, in revised form, September 17, 1973.

AMS (MOS) subject classifications (1970). Primary 18A40, 47A05; Secondary 06A15, 46C10, 46A20.

Key words and phrases. Adjoint, adjoint linear operators, adjoint functors, adjoint on the right, Galois connection, Hilbert space, poset, topological vector space, dual system.

(c) American Mathematical Society 1974 
(ii) If the maps $T_{0}$ and $T_{1}$ are adjoint, then the induced contravariant functors $D_{0}$ and $D_{1}$ are adjoint on the right.

(iii) If the induced contravariant functors $D_{0}$ and $D_{1}$ are adjoint on the right, then there is a scalar $\lambda$ such that $\lambda T_{0}$ and $T_{1}$ are adjoint. (If $T_{0} \neq 0$, then $\lambda$ is unique.)

(iv) Modulo scalars the passage from maps to functors is one-to-one.

We state an immediate consequence.

COROllary. If $T_{0}$ is selfadjoint, then $D_{0}$ is selfadjoint.

2. Adjoints in dual systems. Consider two dual systems $E_{i}, F_{i},(,)_{i}$, each consisting of a pair of vector spaces equipped with a nonsingular bilinear form over a (nondiscrete valuated) field $K$. Consider two linear transformations $T_{0}: E_{0} \rightarrow E_{1}$ and $T_{1}: F_{1} \rightarrow F_{0}$. Let $\mathscr{C}_{0}\left(\mathscr{C}_{1}\right)$ be the small category whose set of objects $\left|\mathscr{C}_{0}\right|\left(\left|\mathscr{C}_{1}\right|\right)$ consists of all subsets of $E_{0}\left(F_{1}\right)$, and whose only arrows are inclusions, for example $X \rightarrow Y$ in $\mathscr{C}_{0}$ iff $X \subseteq Y \subseteq$ $E_{0}$. For $A \in\left|\mathscr{C}_{0}\right|$ and $B \in\left|\mathscr{C}_{1}\right|$, let

and

$$
D_{0} A=\left\{y \in F_{1} \mid\left(T_{0} a, y\right)_{1}=0, \forall a \in A\right\},
$$

$$
D_{1} B=\left\{x \in E_{0} \mid\left(x, T_{1} b\right)_{0}=0, \forall b \in B\right\} .
$$

THEOREM 2. For dual systems, with $T_{i}$ and $D_{i}$ as above, the statements of Theorem 1 hold.

3. Definitions, remarks, and examples. Given a complex Hilbert space $H_{i}$, define a dual system by $E_{i}=H_{i}$ and $F_{i}$ equals $H_{i}$ with the conjugate complex scalar multiplication thereby allowing the bilinear form on $E_{i} \times F_{i}$ to be the given Hermitian inner product on $H_{i}$. Note that a function $T_{1}: H_{1} \rightarrow H_{0}$ is linear iff it is linear as $F_{1} \rightarrow F_{0}$. Thus the functors $D_{i}$ of $\S 2$ generalize those of $\$ 1$.

Recall that the pair of maps $T_{0}$ and $T_{1}$ between Hilbert spaces (dual systems) are adjoint [2, p. 39] $\left(\left[5\right.\right.$, p. 128]) if for all $(x, y)$ in $H_{0} \times H_{1}$ (in $\left.E_{0} \times F_{1}\right)$

$$
\left(T_{0} x, y\right)_{1}=\left(x, T_{1} y\right)_{0},
$$

while two contravariant functors $D_{0}$ and $D_{1}$ are adjoint on the right [1, p. 81] if we have the natural isomorphism of hom-sets

(AF) $\quad \mathscr{C}_{1}\left(Y, D_{0} X\right) \simeq \mathscr{C}_{0}\left(X, D_{1} Y\right), \quad \forall(X, Y) \in\left|\mathscr{C}_{0}\right| \times\left|\mathscr{C}_{1}\right|$.

For Hilbert spaces these are both symmetric relationships (unlike the adjointness of covariant functors). In the special case where $T_{0}=T_{1}=$ the identity map on $H_{0}$, we have $D_{0} A=A^{\perp}$ the orthogonal complement of the subset $A$. 
Since the categories $\mathscr{C}_{0}$ and $\mathscr{C}_{1}$ are essentially posets, having $D_{0}$ and $D_{1}$ adjoint on the right is essentially a Galois connection [4, Theorem 1, p. 93].

All statements and proofs in this note hold if the categories $\mathscr{C}_{i}$ are replaced by their full subcategories whose objects are closed subspaces and if the singleton subset $\{x\}$ is replaced by its span $\{\lambda x \mid \lambda \in K\}$.

For the linear transformation $T_{0}: E_{0} \rightarrow E_{1}$, existence of an adjoint is equivalent to weak continuity [5, p. 128].

Finally, everything in Theorem 1 is a special case of Theorem 2. So it only remains to prove the latter.

4. Proof of the general theorem. (i) Given the arrow $X \rightarrow Y$ in $\mathscr{C}_{0}$, one verifies $D_{0} Y \subseteq D_{0} X \subseteq F_{1}$; similarly, $D_{1}$ is functorial.

(ii) If the maps $T_{0}$ and $T_{1}$ are adjoint, then the equation (AM) holds. We must show (AF) holds. This follows from a reversible chain of implications: $Y \subseteq D_{0} X$ implies $\forall(x, y) \in X \times Y$ that $\left(T_{0} x, y\right)_{1}=0$, which by adjointness (AM) implies $\forall(x, y) \in X \times Y$ that $\left(x, T_{1} y\right)_{0}=0$, hence $X \subseteq D_{1} Y$.

(iii) Given (AF), the left side of (AM) equals 0 iff the right side of (AM) equals 0 , since $\{y\} \subseteq D_{0}\{x\}$ iff $\{x\} \subseteq D_{1}\{y\}$. Thus $T_{0}=0$ implies (AM) holds, since both sides are 0 .

Otherwise pick a vector $x \in E_{0}$ with $T_{0} x \neq 0$, and consider the linear functionals $f=\left(T_{0} x,\right)_{1}$ and $g=\left(x, T_{1}\right)_{0}: F_{1} \rightarrow K$. For $y \in F_{1}, f y=0$ implies $g y=0$ by (AF). Thus there is a unique scalar $\lambda \in K$ with $g=\lambda f$. It remains to show that

$$
\left(\lambda T_{0} a, b\right)=\left(a, T_{1} b\right), \forall(a, b) \in E_{0} \times F_{1} .
$$

The equation holds if $T_{0} a$ is a multiple of $T_{0} x$, since $T_{0} a=\mu T_{0} x$ implies $a-\mu x=n \in \operatorname{ker} T_{0}$, which implies $\left(n, T_{1} b\right)_{0}=0$ by (AF). Otherwise, if $T_{0} a$ and $T_{0} x$ are linearly independent, denote $x^{\prime}=a$ and $x^{\prime \prime}=x^{\prime}+x$ with corresponding linear functionals and scalars as before satisfying $g^{\prime}=$ $\lambda^{\prime} f^{\prime}$ and $g^{\prime \prime}=\lambda^{\prime \prime} f^{\prime \prime}$. Consider the chain of equalities:

$$
\lambda^{\prime} f^{\prime}+\lambda f=g^{\prime}+g=g^{\prime \prime}=\lambda^{\prime \prime} f^{\prime \prime}=\lambda^{\prime \prime} f^{\prime}+\lambda^{\prime \prime} f .
$$

Hence we have $\lambda^{\prime}=\lambda^{\prime \prime}=\lambda$, thus the adjointness equation holds for all $a \in E_{0}$. Note. We have freely used the bilinearity of the bilinear forms and the linearity of the maps, but no continuity or prior existence of adjoints was assumed.

(iv) If the two maps $T_{0}$ and $T_{0}^{\prime}$ induce the same functor $D_{0}$, we will show $T_{0}^{\prime}=\lambda T_{0}$ for some scalar. We may assume $T_{0}^{\prime}$ has an adjoint $T_{1}$ by enlarging $F_{0}$ and $F_{1}$ if necessary [5, p. 128]. Then by (iii), there is $\lambda \in K$ such that $\lambda T_{0}$ is adjoint to $T_{1}$. Hence $T_{0}^{\prime}$ equals $\lambda T_{0}$. 


\section{REFERENCES}

1. P. Freyd, Abelian categories: An introduction to the theory of functors, Harper and Row, New York, 1964.

2. P. Halmos, Introduction to Hilbert spaces, 2nd ed., Chelsea, New York, 1957.

3. S. Mac Lane, The influence of $M$. H. Stone on the origins of category theory, Conference on Functional Analysis and Related Fields, F. E. Browder, Ed., Springer, New York, 1970.

4. - Categories for the working mathematician, Springer, New York, 1971.

5. H. H. Schaefer, Topological vector spaces, Macmillan, New York, 1966. MR 33 \#1689.

Department of Mathematics, University of California, Irvine, California 92664 